\title{
Superconductivity and Charge-Density Wave Phase in the Holstein Model: a Weak Coupling Limit
}

\author{
P. GRzybowski* AND R. Micnas \\ Solid State Theory Division, Institute of Physics, Adam Mickiewicz University \\ Umultowska 85, 61-614 Poznań, Poland
}

Dedicated to Professor Józef Spatek on the occasion of his 60th birthday

The variational canonical transformation method has been applied to the Holstein model to obtain an effective polaronic Hamiltonian, which is subsequently analyzed in the limit of a weak effective electron-electron interaction. A competition between the superconducting and charge-density wave phases has been studied in the light of strong polaronic effects. The phase diagrams illustrating the system evolution from adiabatic to anti-adiabatic limit are presented.

PACS numbers: 71.38.-k, 71.45.Lr, 74.20.Mn

\section{Introduction}

Polaronic superconductivity is one of the candidates for explaining the properties of unconventional superconductors, and as such it has drawn considerable attention $[1,2]$. One of the interesting questions related to this problem concerns the influence of polaronic effects on the competition between superconducting and charge-density wave (CDW) states. The paradigmatic model for studying polaronic systems is the Holstein-Hubbard one [3], which in the standard notation reads

$$
\begin{aligned}
H= & -t \sum_{i, j, \sigma}^{\prime} c_{i \sigma}^{\dagger} c_{j \sigma}+\sum_{i} g\left(a_{i}^{\dagger}+a_{i}\right) n_{i}+\hbar \omega \sum_{i}\left(a_{i}^{\dagger} a_{i}+\frac{1}{2}\right) \\
& +U \sum_{i} n_{i \uparrow} n_{i \downarrow}-\mu \sum_{i} n_{i},
\end{aligned}
$$

where $c_{i \sigma}^{\dagger}, c_{i \sigma}\left(a_{i}^{\dagger}, a_{i}\right)$ are the creation and annihilation operators for electrons (phonons), respectively, and the on-site electron number operator $n_{i}=n_{i \uparrow}+n_{i \downarrow}=$

${ }^{*}$ corresponding author; e-mail: grzyb@amu.edu.pl 
$c_{i \uparrow}^{\dagger} c_{i \uparrow}+c_{i \downarrow}^{\dagger} c_{i \downarrow}$. In the hopping term, the prime excludes $i=j$ and the sum runs over nearest neighbours. It is convenient to introduce the parameters $\bar{g}=g / \hbar \omega$ and $\varepsilon_{\mathrm{p}}=g^{2} / \hbar \omega$ describing the polaronic band narrowing and the energy scale of phonon mediated electron-electron attraction, respectively. The standard theory of electron-phonon systems, due to Migdal [4], consists in a perturbational treatment of the electron-phonon interaction and is valid for weak to moderate electron-phonon coupling $\varepsilon_{\mathrm{p}} / D<0.5$ (where $D$ is the half of the free electron band width) and adiabatic phonons. Alternatively, strong polaronic effects, going beyond the Migdal theory, require large values of $\varepsilon_{\mathrm{p}}$ or non-adiabatic phonons. Additionally, high values of $\varepsilon_{\mathrm{p}}$ may lead to a strong attractive effective electronelectron interaction and the occurrence of strongly bound on-site polaronic pairs (bipolarons). In this contribution however we will analyze the case of attractive but weak effective electron-electron interaction. In this case, the most interesting polaronic effects occur when the phonon energies are comparable to the electron kinetic energy $\hbar \omega \approx t$.

\section{Variational canonical transformations and the effective polaron Hamiltonian}

One of the methods for non-perturbational treatment of the electron-phonon interaction consists of performing variational canonical transformations (VCT), usually modifications of the Lang-Firsov transformation (LFT) [5], followed by averaging over the phonon degrees of freedom in order to obtain an effective polaron Hamiltonian. The pioneering papers of Nasu [6] used VCT to study polaronic effects on the competition between the CDW and superconducting phases. Unfortunately, his form of VCT is not suitable for studying the Holstein-Hubbard model on a bipartite lattice, since it breaks the electron-hole symmetry of the model. The addition of a term describing homogeneous lattice displacement to the generator of the transformation (included, to our knowledge, for the first time in [7]) resolves this problem. In this paper we will use the following VCT, adapted to bipartite lattices:

$$
\begin{aligned}
& \tilde{H}=V^{\dagger} H V, \quad V=\exp \left(\hat{S}_{1}\right) \exp \left(\hat{S}_{2}\right), \\
& \hat{S}_{1}=-(1+\gamma) \bar{g} \sum_{i, \sigma}\left(a_{i}^{\dagger}-a_{i}\right) c_{i \sigma}^{\dagger} c_{i \sigma}-\gamma_{0} \bar{g} \sum_{i}\left(a_{i}^{\dagger}-a_{i}\right)-\Delta_{0} \bar{g} \sum_{i}\left(a_{i}^{\dagger}-a_{i}\right) R_{i}, \\
& \hat{S}_{2}=-r \sum_{i}\left(a_{i}^{\dagger^{2}}-a_{i}^{2}\right),
\end{aligned}
$$

where $R_{j}$ takes the value +1 on one of the sublattices and -1 on the other. The variational parameters $\gamma_{0}, \gamma$, and $\Delta_{0}$ describe the homogeneous, modified Lang-Firsov and staggered lattice displacements, respectively. The variational parameter $r$ describes squeezing of the phonon modes [8]. The parameter $\gamma$ takes the values from the range $\langle-1,0\rangle$, with $\gamma=0$ corresponding to the complete Lang-Firsov lattice displacement. The transformed Hamiltonian $\tilde{H}$ is then averaged over the phonon 
degrees of freedom using the free-phonon density matrix

$$
\rho_{0}=\exp \left(-\beta H_{\mathrm{ph}}\right) / \operatorname{Tr}\left(\exp \left(-\beta H_{\mathrm{ph}}\right)\right),
$$

where $H_{\mathrm{ph}}=\hbar \omega \sum_{i}\left(a_{i}^{\dagger} a_{i}+\frac{1}{2}\right)$ and $\beta=\frac{1}{k_{\mathrm{B}} T}$. This leads to the following effective polaronic Hamiltonian:

$$
\begin{aligned}
H_{\mathrm{eff}} & =\operatorname{Tr}\left(\rho_{0} \tilde{H}\right)+\frac{1}{\beta} \operatorname{Tr}\left(\rho_{0} \ln \rho_{0}\right)=-t \eta \sum_{i, j, \sigma}^{\prime} c_{i \sigma}^{\dagger} c_{j \sigma}+\bar{U} \sum_{i} c_{i \uparrow}^{\dagger} c_{i \uparrow} c_{i \downarrow}^{\dagger} c_{i \downarrow} \\
& +2 \varepsilon_{\mathrm{p}} \gamma \Delta_{0} \sum_{i, \sigma} R_{i} c_{i \sigma}^{\dagger} c_{i \sigma}-\bar{\mu} \sum_{i, \sigma} c_{i \sigma}^{\dagger} c_{i \sigma}+N \varepsilon_{\mathrm{p}} \gamma_{0}^{2}+N \varepsilon_{\mathrm{p}} \Delta_{0}^{2}+\frac{N \hbar \omega}{2} \\
& \times[\cosh (4 r)-1] \operatorname{coth}\left(\frac{\beta \hbar \omega}{2}\right)+\frac{N \hbar \omega}{2} \ln \left(\sinh \left(\frac{\beta \hbar \omega}{2}\right)\right) /\left(\frac{\beta \hbar \omega}{2}\right),
\end{aligned}
$$

where

$$
\begin{aligned}
& \eta=\exp \left(-\bar{g}^{2}(1+\gamma)^{2} \exp (-4 r) \operatorname{coth}\left(\frac{\beta \hbar \omega}{2}\right)\right), \\
& \bar{U}=U-2 \varepsilon_{\mathrm{p}}\left(1-\gamma^{2}\right), \quad \bar{\mu}=\mu+\varepsilon_{\mathrm{p}}\left(1-\gamma^{2}-2 \gamma \gamma_{0}\right) .
\end{aligned}
$$

The Hamiltonian (4) can be instantly minimized with respect to the variational parameter $\gamma_{0}$ yielding

$$
\gamma_{0}=-\gamma \frac{1}{N} \sum_{i, \sigma} c_{i \sigma}^{\dagger} c_{i \sigma}=-\gamma n
$$

which gives a shift to the chemical potential: $\bar{\mu}=\mu+\varepsilon_{\mathrm{p}}\left[1+(2 n-1) \gamma^{2}\right]$, where $n$ is the electron density. The effective polaronic Hamiltonian (4) is of the form of the Hubbard model with an additional alternating on-site energy term $2 \varepsilon_{\mathrm{p}} \gamma \Delta_{0} \sum_{i, \sigma} R_{i} c_{i \sigma}^{\dagger} c_{i \sigma}$. The polaron hopping integral is reduced with respect to the bare one by the band narrowing factor $\eta$. The procedure described above has to be modified in the limit of the on-site bipolarons formation. In such a case, the averaging over the phonon subsystem should be performed after obtaining the effective bipolaronic Hamiltonian. Therefore, in this paper we constrain our analysis to the case when the effective Hubbard interaction $\bar{U}$ is attractive and weak: $\bar{U}<2 z t \eta$ ( $z$ is the number of nearest neighbours).

\section{Mean-field equations}

We analyze Hamiltonian (4) in the framework of the Hartree-type mean-field approximation (MFA). Following [9], the MFA equations will be derived using the Bogolyubov variational principle. We consider pure phases: non-ordered (NO), "s-wave" superconducting (SC) and commensurate CDW with the vector $\boldsymbol{Q}=$ $(\pi / a, \pi / a, \ldots)$. The trial Hamiltonian $H_{0}$ reads

$$
\begin{aligned}
H_{0}= & \sum_{\boldsymbol{k}, \sigma}\left(\eta \epsilon_{k}-\bar{\mu}-A_{0}\right) c_{\boldsymbol{k} \sigma}^{\dagger} c_{\boldsymbol{k} \sigma}-\frac{1}{2} \sum_{\boldsymbol{k}, \sigma}\left(\Delta c_{\boldsymbol{k} \sigma}^{\dagger} c_{\boldsymbol{k}+\boldsymbol{Q} \sigma}+\text { h.c. }\right) \\
& +\frac{1}{2} \sum_{\boldsymbol{k}, \sigma}\left(X c_{\boldsymbol{k} \sigma}^{\dagger} c_{-\boldsymbol{k}-\sigma}^{\dagger}+\text { h.c. }\right)
\end{aligned}
$$


where $A_{0}, \Delta$, and $X$ are variational parameters. The parameters $\Delta$ and $X$ represent the energy gaps for CDW and SC phases, respectively, whereas $A_{0}$ describes a chemical potential shift. Diagonalization of the trial Hamiltonian (7), with the use of the perfect nesting condition $\epsilon_{\boldsymbol{k}+\boldsymbol{Q}}=-\epsilon_{k}$, yields the quasiparticle energies $\left( \pm A_{\boldsymbol{k}}^{+}\right)$and $\left( \pm A_{\boldsymbol{k}}^{-}\right)$, where

$$
\begin{aligned}
& A_{\boldsymbol{k}}^{ \pm}=\sqrt{\left(E_{\boldsymbol{k}} \pm \overline{\bar{\mu}}\right)^{2}+X^{2}}, \\
& E_{\boldsymbol{k}}=\sqrt{\eta^{2} \epsilon_{\boldsymbol{k}}^{2}+\Delta^{2}}, \\
& \overline{\bar{\mu}}=\bar{\mu}+A_{0}=\mu+\varepsilon_{\mathrm{p}}\left[1+(2 n-1) \gamma^{2}\right]+A_{0} .
\end{aligned}
$$

The variational free energy is

$$
\begin{aligned}
\frac{F_{0}}{N}= & -\frac{1}{\beta N} \sum_{\boldsymbol{k}, \sigma}\left[\ln \left(2 \cosh \left(\frac{\beta}{2} A_{\boldsymbol{k}}^{+}\right)\right)+\ln \left(2 \cosh \left(\frac{\beta}{2} A_{\boldsymbol{k}}^{-}\right)\right)\right]+(n-1) \overline{\bar{\mu}} \\
& +\bar{U}\left(n^{2}+n_{Q}^{2}+4 x_{0}^{2}\right) / 4+\varepsilon_{\mathrm{p}} \Delta_{0}^{2}+\left(\Delta+2 \varepsilon_{\mathrm{p}} \gamma \Delta_{0}\right) n_{Q}+2 X x_{0} \\
& -n \varepsilon_{\mathrm{p}}\left[1+(2 n-1) \gamma^{2}\right]+\frac{\hbar \omega}{2}[\cosh (4 r)-1] \operatorname{coth}\left(\frac{\beta \hbar \omega}{2}\right) \\
& +\frac{\hbar \omega}{2} \ln \left(2 \sinh \left(\frac{\beta \hbar \omega}{2}\right)\right) /\left(\frac{\beta \hbar \omega}{2}\right),
\end{aligned}
$$

where $n_{Q}$ and $x_{0}$ are the CDW and SC order parameters, respectively, given by the equations

$$
\begin{aligned}
n_{Q} & =\frac{1}{N} \sum_{\boldsymbol{k}, \sigma}\left\langle c_{\boldsymbol{k}+\boldsymbol{Q} \sigma}^{\dagger} c_{\boldsymbol{k} \sigma}\right\rangle_{0} \\
& =\frac{\Delta}{2 N} \sum_{\boldsymbol{k}}\left[\left(1+\frac{\overline{\bar{\mu}}}{E_{\boldsymbol{k}}}\right) B_{\boldsymbol{k}}^{+}+\left(1-\frac{\overline{\bar{\mu}}}{E_{\boldsymbol{k}}}\right) B_{\boldsymbol{k}}^{-}\right] \\
x_{0} & =\frac{1}{2 N} \sum_{\boldsymbol{k}, \sigma}\left\langle c_{\boldsymbol{k} \sigma} c_{-\boldsymbol{k}-\sigma}\right\rangle_{0}=\frac{X}{4 N} \sum_{\boldsymbol{k}}\left(B_{\boldsymbol{k}}^{+}+B_{\boldsymbol{k}}^{-}\right), \\
n= & \frac{1}{N} \sum_{\boldsymbol{k}, \sigma}\left\langle c_{\boldsymbol{k} \sigma}^{\dagger} c_{\boldsymbol{k} \sigma}\right\rangle_{0}=1+\frac{1}{2 N} \sum_{\boldsymbol{k}}\left[\left(E_{\boldsymbol{k}}+\overline{\bar{\mu}}\right) B_{\boldsymbol{k}}^{+}-\left(E_{\boldsymbol{k}}-\overline{\bar{\mu}}\right) B_{\boldsymbol{k}}^{-}\right],
\end{aligned}
$$

where

$$
B_{k}^{ \pm}=\left(A_{k}^{ \pm}\right)^{-1} \tanh \left(\frac{\beta}{2} A_{k}^{ \pm}\right)
$$

The minimization of (9) with respect to $\Delta, X$, and $A_{0}$, leads to the self-consistent integral equations

$$
\begin{aligned}
& \Delta=-\bar{U} n_{Q} / 2-2 \varepsilon_{\mathrm{p}} \gamma \Delta_{0}, \\
& A_{0}=-\bar{U} n / 2, \\
& X=-\bar{U} x_{0} .
\end{aligned}
$$

Additional minimization of (9) with respect to $\Delta_{0}$ yields $\Delta_{0}=-\gamma n_{Q}$ which allows a few simplifications. Introducing 


$$
\overline{\bar{U}}=\bar{U}-4 \varepsilon_{\mathrm{p}} \gamma^{2}=U-2 \varepsilon_{\mathrm{p}}\left(1+\gamma^{2}\right)
$$

we find $\Delta_{0}=2 \gamma \Delta / \overline{\bar{U}}$. The equation for the gap function $\Delta$ can be now recast into a more familiar form

$$
\Delta=-\overline{\bar{U}} n_{Q} / 2 \text {. }
$$

Equation (9) can also be written in a form of the mean-field free energy for the attractive Hubbard model [9]

$$
\begin{aligned}
\frac{F_{0}}{N}= & -\frac{1}{\beta N} \sum_{\boldsymbol{k}, \sigma}\left[\ln \left(2 \cosh \left(\frac{\beta}{2} A_{\boldsymbol{k}}^{+}\right)\right)+\ln \left(2 \cosh \left(\frac{\beta}{2} A_{\boldsymbol{k}}^{-}\right)\right)\right]+(n-1) \overline{\bar{\mu}} \\
& +\frac{\overline{\bar{U}} n^{2}}{4}-\frac{\Delta^{2}}{\overline{\bar{U}}}-\frac{X^{2}}{\bar{U}}-\varepsilon_{\mathrm{p}}\left(1-\gamma^{2}\right) n+\frac{\hbar \omega}{2}[\cosh (4 r)-1] \operatorname{coth}\left(\frac{\beta \hbar \omega}{2}\right) \\
& +\frac{\hbar \omega}{2} \ln \left(2 \sinh \left(\frac{\beta \hbar \omega}{2}\right)\right) /\left(\frac{\beta \hbar \omega}{2}\right) .
\end{aligned}
$$

The main difference is that CDW and SC phases in Eq. (17) experience different strengths of the effective Hubbard interaction $U_{\text {eff }}$, as is also evident from the self-consistent Eqs. (14c) and (16). In the SC phase $U_{\text {eff }}=\bar{U}$, whereas for the CDW phase $U_{\text {eff }}=\overline{\bar{U}}$. Comparison of (5b) and (15) leads to a conclusion that $|\overline{\bar{U}}| \geq|\bar{U}|$, which is in agreement with the well-known fact that for $n=1$ the CDW phase is more stable than the SC phase in the Holstein model.

Of course, the free energy in Eq. (17) has also to be minimized with respect to the parameters $\gamma$ and $r$, so the self-consistent equations for $\Delta, X$, and $A_{0}$ have to be solved along with the conditions $\partial F_{0} / \partial \gamma=0$ and $\partial F_{0} / \partial r=0$. The solution of this set of equations is obtained numerically.

\section{Results and discussion}

In this section we present numerical results for the pure Holstein model $(U=0)$ on setting $D=1$ as the energy unit. We analyze the $2 \mathrm{D}$ square lattice case, using either the exact or the rectangular density of states (DOS).

\subsection{Ground state phase diagrams}

We focus on the system evolution from the anti-adiabatic to the adiabatic regime for a constant value of the electron-phonon coupling $g$, in the ground state. In Fig. 1 and Fig. 2 we present the ground state phase diagrams for some representative cases. The uniform CDW phase exists only for $n=1$. In the antiadiabatic regime and for low electron densities, the system is in the $\mathrm{SC}$ phase. The crossover from BCS to the Bose-Einstein condensate (BEC) superconductivity is estimated with the help of the Leggett criterion [10] that the chemical potential reaches the lower band edge: $\overline{\bar{\mu}}=-\eta D$. In Fig. 1 this crossover occurs in extremely diluted limit $n \rightarrow 0$. For comparison we show the results for the BCSBEC crossover obtained when the variational canonical transformations of the form (2) were replaced by the ordinary Lang-Firsov transformation $\left(\gamma=0, \gamma_{0}=0\right.$, 


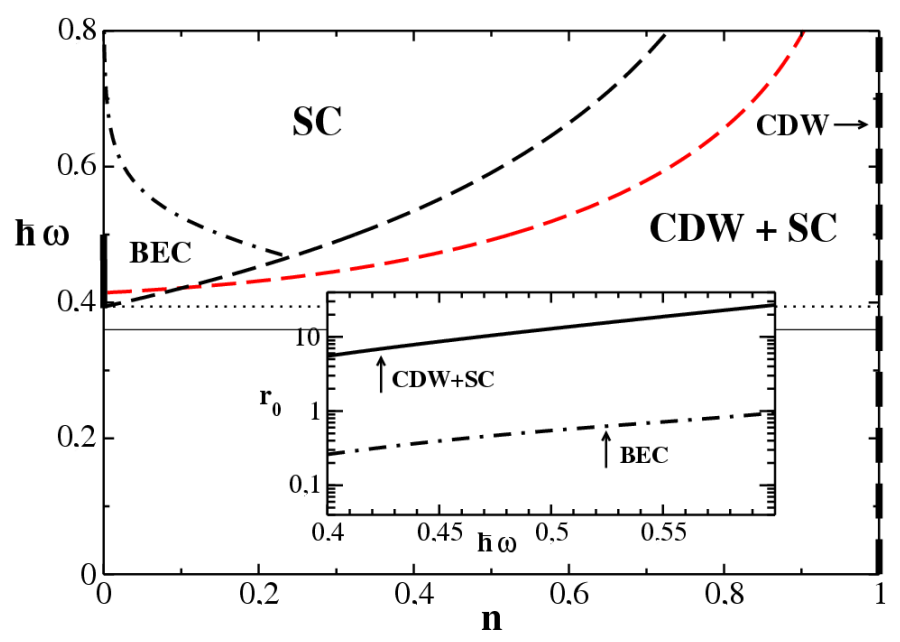

Fig. 1. Ground state phase diagram for $g=0.45$. All black lines are obtained with the exact DOS, a coloured line is obtained with the rectangular DOS for comparison. First-order transitions from the phase separated states to the pure SC and CDW phases are shown as thick dashed lines. The BCS to BEC crossover, in the present case, is denoted by thick solid line, while in the case of ordinary LFT by dot-dashed line. Inset shows the pair radius $r_{0}$ (in units of lattice spacings $a$ ) and its dependence on $\hbar \omega$ for $n=0.1$. The case of LFT is denoted by dot-dashed line.

$\left.\Delta_{0}=0, r=0\right)$. In this case the BCS-BEC crossover occurs also for larger electron densities. To address these differences we estimated the superconducting pairs radii $r_{0}$ using the formula from Ref. [11]. The results for $n=0.1$ are shown in the inset. As can be seen, the calculations with variational canonical transformations of the form (2) lead to much larger pairs than the calculations with ordinary LangFirsov transformation, which ignore the effects of the phonon adiabaticity. As a result, the BCS-BEC crossover, in the case of the VCT approach used in this paper, is density driven with bound but large polaron pairs in the BEC limit. In contrast, the ordinary LFT approach results in the BCS-BEC crossover driven by a small pair radius with on-site bipolarons in the BEC limit.

For electron densities close to half-filling we obtain the CDW-SC phase separated (PS) state which for sufficiently low values of $\hbar \omega$ converts into the complete phase separated (CPS) state (with a fraction of the system in the CDW phase with the charge density $n_{\mathrm{CDW}}=1$ and with the rest of the system with zero charge density). In Fig. 1 we indicate the transition to the CPS state by a thin dotted line below which the superconductivity vanishes. We add that a possible mixed solution with both $x_{0} \neq 0$ and $n_{Q} \neq 0$ exists but has a higher free energy than PS state for the parameters studied in this paper and the rectangular DOS. One should also consider a possibility of existence of incommensurate CDW phase (ICDW), at least close to half-filling. To conclude which of the two states: PS or 
ICDW will realize in real systems one would have to include long-range Coulomb forces into the model considered (1). The latter remark is valid for all other cases of phase separated states discussed in this paper.

On approaching a small $\hbar \omega$ limit with a constant value of $g$, the phonon mediated electron-electron attraction becomes large and the system crosses to the regime of on-site bipolarons. Since we have constrained our analysis to the polaronic regime, the following qualitative criterion may serve as condition for the crossover between these regimes:

$$
2 \eta D=U_{\text {eff }} .
$$

We solved the condition (18) for the uniform CDW phase at $n=1$, since this phase experiences stronger $U_{\text {eff }}=\overline{\bar{U}}$ and dominates in the phase diagram in the adiabatic limit. The regions of bipolaronic and polaronic character of the CDW phase for $n=1$ are shown in the inset of Fig. 2. The dotted line denotes solutions of $\varepsilon_{\mathrm{p}} / D=0.5$. Below this line, in the limit of adiabatic phonons, the Migdal theory is valid. As can be seen in that limit the conditions $(18)$ and $\varepsilon_{\mathrm{p}} / D=0.5$ are equivalent. The inclusion of non-zero repulsive Hubbard $U$ will, however, change this coincidence, shifting the line of the crossover to higher values of $g$. In the ground state diagrams (Figs. 1 and 2) the crossover from polarons to on-site bipolarons is denoted by a thin solid line. Below this line the calculations based on the strong coupling perturbation expansion around the on-site bipolarons limit should be more appropriate. Nevertheless, the results of the perturbation expansion in the adiabatic limit generally support the conclusion that in this limit

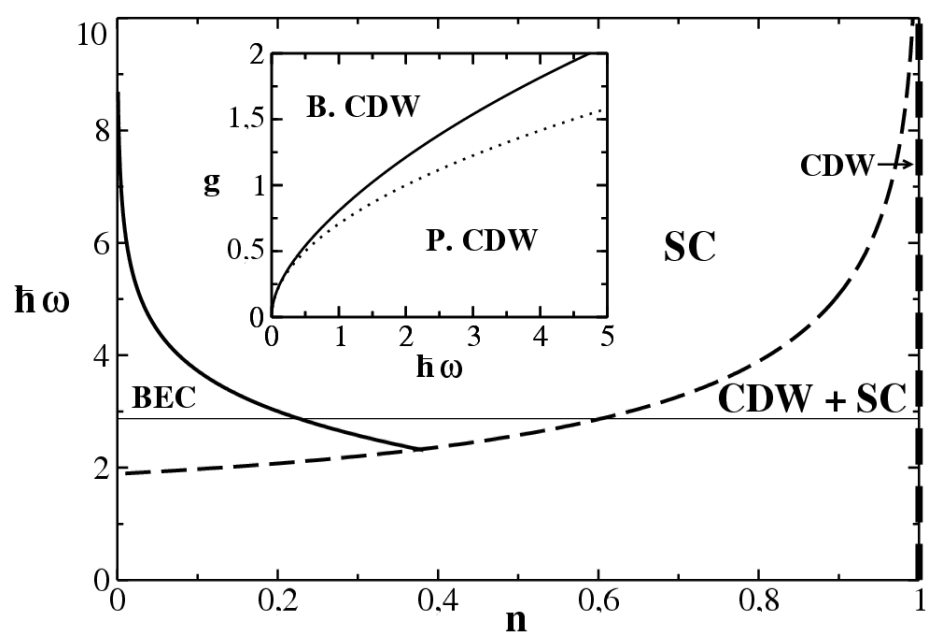

Fig. 2. Ground state phase diagram for $g=1.5$ obtained with the rectangular DOS. First-order transitions from the $\mathrm{CDW}+\mathrm{SC}$ phase separated state to the pure SC and CDW phases are shown as thick dashed lines. The BCS to BEC crossover is denoted by thick solid line. Inset shows the crossover from the polaronic CDW (P. CDW) to the bipolaronic CDW (B. CDW) phase by solid line for $n=1$, obtained with the exact DOS. The solutions of $\varepsilon_{\mathrm{p}} / D=0.5$ are denoted by dotted line. 
the SC phase is practically absent and the ground state diagrams are dominated by the CDW phase. A comparison of Figs. 1 and 2 shows that an increase in electronphonon coupling $g$ shifts the phase boundaries towards higher values of $\hbar \omega$. The effects of the phonon adiabaticity are therefore less important for high values of $g$. This can be observed in the BCS-BEC crossover which becomes similar to that obtained using the ordinary LFT, with a small pair radius. As a result, the crossover to the bipolaronic limit happens already in the SC phase.

\subsection{Temperature phase diagrams}

In the analysis of the CDW-SC competition at $T \neq 0$ we choose $g=0.45$ and $\hbar \omega=0.5$ as a representative set of parameters. Figures 3 and 4 present the temperature phase diagrams for those parameters in the $T-\mu$ and $T-n$ planes, respectively. In Fig. 3 the $\mathrm{SC}-\mathrm{CDW}$ transition is discontinuous. This results in the phase separated $\mathrm{CDW}+\mathrm{SC}$ state in the $T-n$ plane (Fig. 4). For higher temperatures, the $\mathrm{SC}$ phase is replaced by the NO phase (the SC-NO transition is always continuous). As follows from Fig. 3, the CDW to NO transition is continuous for higher temperatures and discontinuous for lower, with a tricritical point (TCP) separating these two types of transition. In Fig. 4 the CDW phase dominates at half-filling, whereas the pure SC phase is restricted to low electron densities. Additionally, one can see the region of $\mathrm{CDW}+\mathrm{NO}$ phase separated state. The presented phase diagrams were obtained for the exact DOS (black lines) as well as the rectangular DOS (coloured lines). In both cases the results are qualitatively the same.

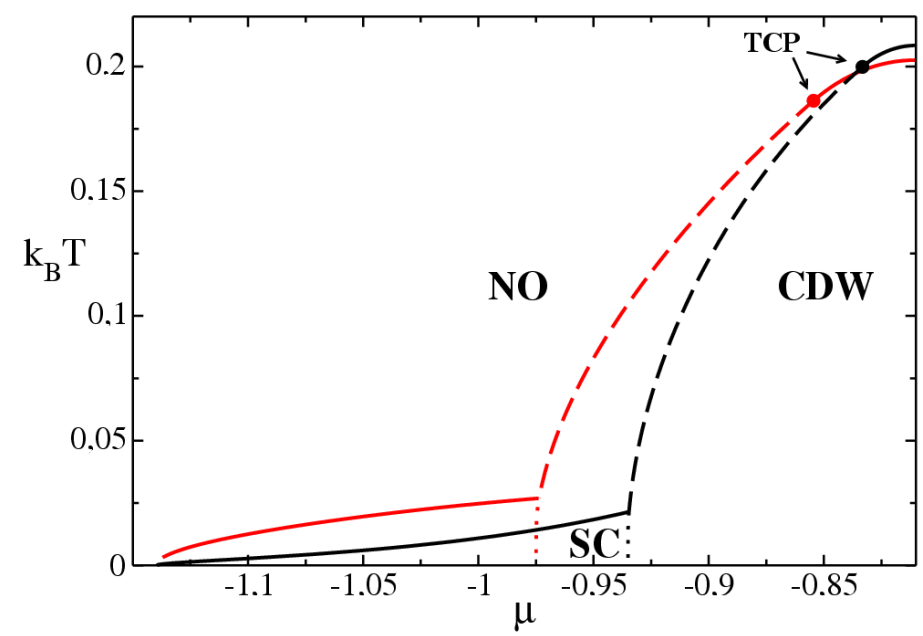

Fig. 3. The $T-\mu$ phase diagram for $g=0.45$ and $\hbar \omega=0.5$. Black and coloured lines are obtained with the exact and rectangular DOS, respectively. Second-order CDW to NO and SC to NO transitions are indicated by solid lines. First-order CDW to NO and CDW to SC transitions are indicated by dashed and dotted lines, respectively. Black dot indicates the tricritical point (TCP). 


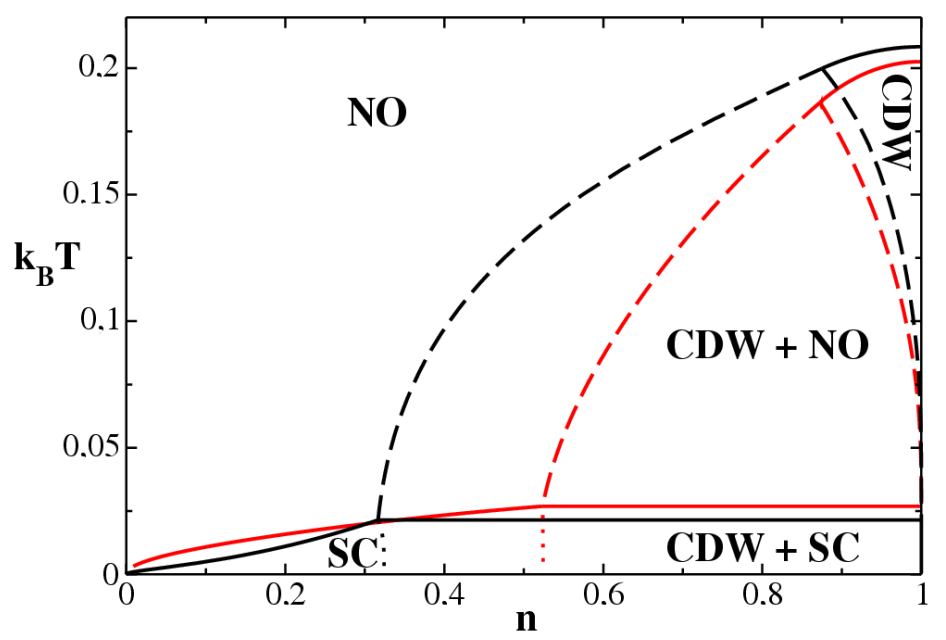

Fig. 4. The $T-n$ phase diagram for $g=0.45$ and $\hbar \omega=0.5$. Black and coloured lines are obtained with the exact and rectangular DOS, respectively. Second-order CDW to NO and SC to NO transitions are indicated by solid lines. First-order transitions to $\mathrm{CDW}+\mathrm{NO}$ and $\mathrm{CDW}+\mathrm{SC}$ phase separated states are indicated by dashed and dotted lines, respectively.

For high values of the phonon frequencies, the Holstein model approaches the attractive Hubbard model, for which the SC dominates for all $n$, with the exact CDW and SC degeneracy at half-filling. It can be seen for example in Fig. 5 and Fig. 6 plotted for $g=1.5$ and $\hbar \omega=5$. In Fig. 5, due to the extension of the region of the $\mathrm{SC}$ phase, the line of the discontinuous CDW to NO transition vanishes, instead we have a bicritical point. As a consequence in Fig. 6 there is no more a $\mathrm{CDW}+\mathrm{NO}$ phase separated state.

Generally, the obtained phase diagrams resemble those of the so-called " $t-U-W$ " or extended Hubbard model [12]. A difference lies in the fact that the effective values of $t, U$, and " $W$ " in the present treatment depend on the variational phonon parameters, and change with temperature or from one phase to another.

Our analysis does not include thermal and phase fluctuation effects and thus the obtained MFA phase diagrams in 2D, especially at finite temperatures, should be treated qualitatively. The SC to NO transition can only occur as a Kosterlitz-Thouless transition. On the other hand, CDW long-range order is possible for $T \neq 0$, however the MFA overestimates the critical temperature of the CDW-NO transition. The fluctuations will particularly influence BCP in Fig. 5. In quasi-2D case, the $\mathrm{BCP}$ will move to lower temperatures bending continuous transition lines.

The numerical results discussed here concern only the pure Holstein model $(U=0)$. A more realistic approach should include a finite repulsive Hubbard 


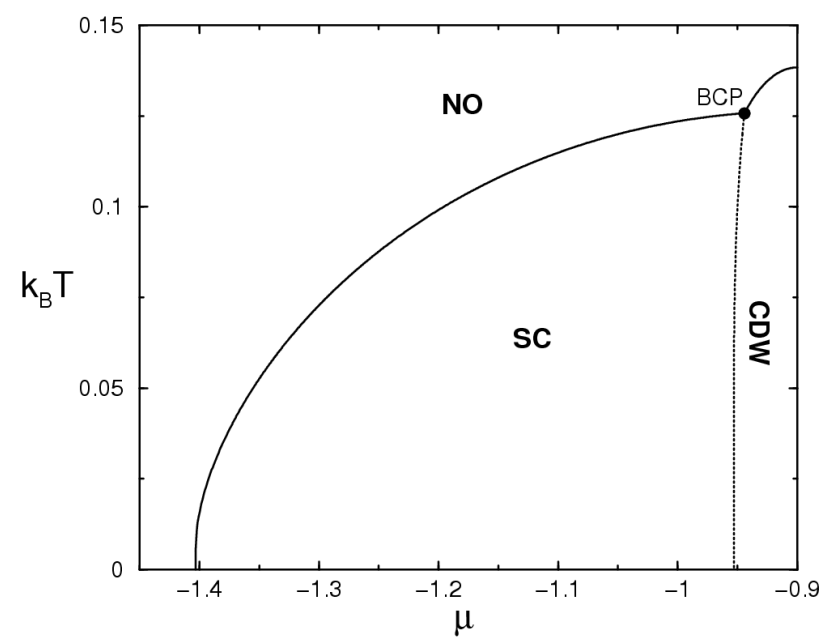

Fig. 5. Phase diagram for $g=1.5$ and $\hbar \omega=5$ in the $T-\mu$ plane, obtained with the rectangular DOS. Second-order CDW to NO and SC to NO transitions are indicated by solid lines. First-order CDW to SC transition is indicated by dotted line. Black dot shows the bicritical point (BCP).

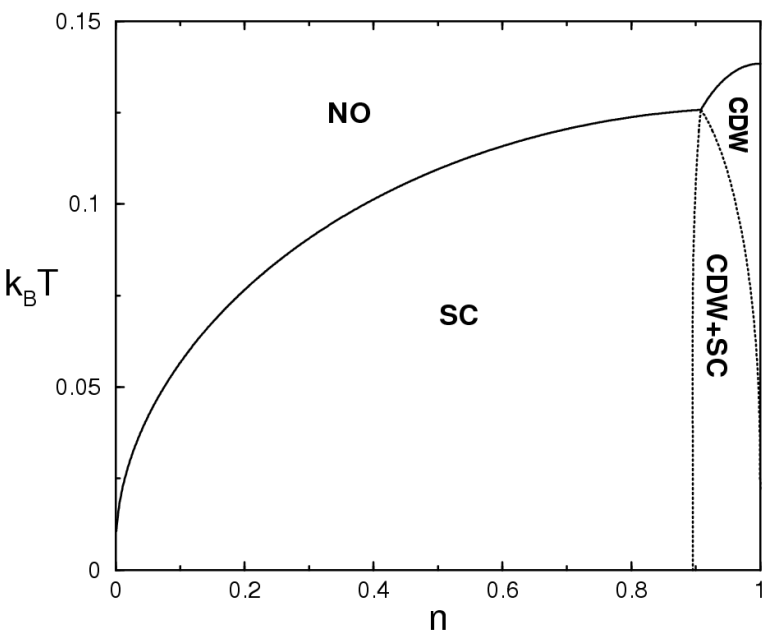

Fig. 6. Phase diagram for $g=1.5$ and $\hbar \omega=5$ in the $T-n$ plane, obtained with the rectangular DOS. Second-order CDW to NO and SC to NO transitions are indicated by solid lines. First-order transitions to the CDW+SC phase separated state are indicated by dotted lines.

interaction $U$. However, our results should remain qualitatively unchanged as long as the effective electron-electron interaction remains weak and attractive. Additionally, even for $U$ slightly exceeding the phonon mediated electron-electron attraction $U_{\mathrm{ph}}$, the existence of bound polaron pairs can occur due to retardation 
effects on $U_{\mathrm{ph}}$, in the dilute limit [13]. In such a case one may expect the existence of the SC phase in a dilute limit, while at larger electron densities one should extend our analysis by considering magnetic phases, especially the SDW phase.

\section{Conclusions}

In this paper we studied a competition between SC and CDW phases in the presence of strong polaronic effects, including the quantum lattice fluctuations. We have presented the ground state as well as finite temperature phase diagrams for the Holstein model in the MFA, for representative sets of the system parameters values. We have found various continuous and discontinuous phase transitions, as well as multicritical points. It is one of the first such a detailed study of this problem in the variational approach. An extended version of this work will be published elsewhere.

\section{Acknowledgments}

This work was supported by the Foundation for Polish Science (FNP) and the State Committee for Scientific Research project No. 1 P03B 08426. We thank R.W. Chhajlany for comments on the manuscript.

\section{References}

[1] R. Micnas, J. Ranninger, S. Robaszkiewicz, Rev. Mod. Phys. 62, 113 (1990).

[2] A.S. Alexandrov, N.F. Mott, Rep. Prog. Phys. 57, 1197 (1994).

[3] T. Holstein, Ann. Phys. (New York) 8, 325 (1959).

[4] G.M. Migdal, Zh. Eksp. Teor. Fiz. 38, 966 (1960) [Sov. Phys. JETP 11, 696 (1960)].

[5] I.G. Lang, Y.A. Firsov, Zh. Eksp. Teor. Fiz. 43, 1843 (1963) [Sov. Phys. JETP 16, 1301 (1963)].

[6] K. Nasu, J. Phys. Soc. Jpn. 54, 1933 (1985); Phys. Rev. B 35, 1748 (1987).

[7] H. Fehske, D. Ihle, J. Loos, U. Trapper, H. Büttner, Z. Phys. B, Condens. Matter 94, 91 (1994).

[8] Zheng Hang, Phys. Rev. B 38, 11865 (1988).

[9] S. Robaszkiewicz, R. Micnas, K.A. Chao, Phys. Rev. B 24, 4018 (1981); 26, 3915 (1982).

[10] A.J. Leggett, Modern Trends in the Theory of Condensed Matter, Springer-Verlag, Berlin 1980, p. 13.

[11] M. Bạk, R. Micnas, J. Phys., Condens. Matter 10, 9029 (1998). Let us note that $L_{1}$ is misprinted and should read $\int \bar{\epsilon}^{2} \rho_{1}(\epsilon) / E^{6}(\epsilon) \mathrm{d} \epsilon$.

[12] B. Tobijaszewska, Ph.D. Thesis, Adam Mickiewicz University, Poznań 2001; S. Robaszkiewicz, G. Pawłowski, Acta Phys. Pol. A 90, 569 (1996); M. Bạk, Acta Phys. Pol. A 106, 637 (2004).

[13] A. Macridin, G.A. Sawatzky, M. Jarrell, Phys. Rev. B 69, 245111 (2004). 